\title{
Development of prophylactic treatment in retinal surgery
}

\author{
JAMES R. HUDSON \\ Moorfields Eye Hospital, High Holborn, London, W.C.I
}

Contributed by request and dedicated to Sir Stewart Duke-Elder

As the years pass, medical science is moving gradually away from the principle of curing disease towards the greater achievement of its prevention. To further this end, community health services are being developed, and pilot studies have already been undertaken in the screening of the population to assist in the early diagnosis of a number of conditions, among them glaucoma. The increased specialization of post-graduate training is providing greater expertise in retinal diagnosis and treatment, and there have been, particularly during the last two decades, substantial advances in the recognition of vitreous and retinal changes which predispose to the development of retinal detachments.

More than a century ago, Coccius (1853) described retinal breaks, but it was not until 67 years later that Gonin (1920) correctly interpreted their significance. The observation that retinal breaks were the pathological lesions that led to retinal detachments inspired many workers to investigate the methods by which the breaks could be sealed and choroidoretinal apposition restored. Weve (1930) introduced diathermy as an alternative to electrocautery or chemical cauterization. At the Fourteenth International Ophthalmological Congress in Madrid, Lindner (1933a) discussed the possibility of preventing retinal detachment. He prophesied that one day an operative method would be developed to achieve this. In the same year Bietti (1933) described the use of cryotherapy in the treatment of retinal detachment, a discovery that was to be overlooked until Cooper ( 1962 ) described the principles and rationale of cryogenic surgery, and outlined the use of a cryotherapy instrument using liquid nitrogen as a coolant. Lincoff and McLean (I965) saw a future for this method of producing choroido-retinal reaction in retinal surgery, and published their observations after animal experiments and clinical trials. Major advances have since been made in instrumentation, and both simple and sophisticated instruments are now available. Examples of these are the Amoils cryosurgical unit (Amoils, 1966) and the Amoilette which uses either nitrous oxide or carbon dioxide (Fison and Wright, 1968).

Between the original description of cryotherapy and its widespread application there were numerous developments in surgical technique, beginning with scleral surgery. Fullthickness scleral resection (Müller, I903; Lindner, I933b) was succeeded by the lamellar procedure (Shapland, I95I, I952), and the latter has remained in use until recently. Indeed, there are still indications for this method. Episcleral implants or implants introduced into scleral pockets began with the use of polyviol materials by Custodis (I95I) and of preserved sclera by Paufique (1963). A number of natural and synthetic materials have subsequently been described, those most commonly used at the present time being silicone rubber and silicone rubber sponge. Encircling techniques began with the Arruga 
suture (Arruga, 1958), which was followed by the use of polyethylene tubing (Schepens, I958), and later by silicone rubber rods or bands. The development of the technique of internal tamponnage using donor vitreous or alternative fluids owes a great deal to the pioneer work of Shafer, Cibis, Hruby, and Balazs.

One of the most important contributions has been the development of the light coagulator by Meyer-Schwickerath (1949). Here at last was a method of inducing choroidoretinal adhesion without the risk of scleral damage, and with the possibility of direct visualization and treatment of the retinal lesion by the same instrument. The ruby laser (Ingram, 1964) and the argon laser (Flocks, 1966), which have similar facilities, have subsequently been developed. The ruby laser provides the means of producing a discrete lesion, but has the disadvantage of having the same spectral value as blood, thereby minimizing its effect on areas of haemorrhage or upon blood vessels. The argon laser, in the green range of the spectrum, is absorbed, and has the additional significant advantage of control of the applications by slit-lamp biomicroscopy.

Along with improvements in operative techniques, there have been parallel developments in methods of examination. The direct ophthalmoscope, which was for so many years the preferred instrument in use in the United Kingdom, has been supplemented, and many would say completely replaced by, the binocular indirect ophthalmoscope, an important contribution by Schepens (1958) to the diagnostic examination of the retina. Its use with indentation techniques has brought more of the peripheral retina within the range of the observer. Biomicroscopy of the peripheral retina and of the vitreous have become possible thanks to slit-lamp microscopy, using the Goldmann 3-mirror contact lens.

Methods of examination and techniques of treatment have advanced together to such an extent that the diagnosis of retinal lesions predisposing to the development of a detachment has become, omitting exceptional cases, a matter of routine. Their control by modern methods of inducing choroido-retinal adhesion, and of minimizing the effects of vitreous traction, has become the rule rather than the exception.

The present field of progress in the prophylactic treatment of retinal detachments is therefore concerned with the correct interpretation of the clinical findings, and with the choice of technique for their management.

Lincoff ( $196 \mathrm{r}$ ) has discussed the relative dangers of different types of retinal tears and holes. Other authors have assessed the prognostic significance of the many pathological changes which are found on retinal examination, and have tried to distinguish between dangerous and innocuous conditions. One of the best expositions appears in an editorial written for the Archives of Ophthalmology by Cockerham and MacKenzie Freeman (1968), who discussed the relevance of choroido-retinal lesions in relation to their predisposition to cause retinal detachments. They emphasized that the presence of choroido-retinal lesions, and even of retinal breaks, is not necessarily an indication for their treatment.

"Many common ocular fundus lesions", they say, "require no treatment. These include "whitewith-pressure', 'white-without-pressure', peripheral cystoid degeneration, chorioretinal degeneration, peripheral chorioretinal atrophy (also known as pavingstone or cobblestone degeneration), cysts of the pars plana ciliaris, and meridional retinal folds. Other common but equally innocuous peripheral fundus lesions which simulate retinal breaks are ora bays isolated in the retina and tags of granular tissue. Small retinal breaks near the ora serrata which tend to be single require no treatment, especially when found in young patients. Suspected macular holes are usually partial thickness defects or cysts that do not progress to retinal detachment and should not be treated prophylactically, even though they have the appearance of holes with ophthalmoscopy and slit-lamp microscopy.

"Senile retinoschisis is usually stationary or very slowly progressive and requires no treatment. 
However, if progression endangers the macula or is documented by accurate drawings and measurements of visual fields, or if breaks develop in the outer layer of the retinoschisis cavity, therapy may be considered.

"Treatment of lattice degeneration should be considered in three situations: when the ocular fundus is extensively involved or shows lesions which are proven to be progressive, especially superiorly and temporally; when retinal breaks are present along the edge of the lattice area; and when vitreous traction, indicated by visible vitreous membranes attached to the elevated margins of the lattice, is present.

"Retinal breaks with minimal retinal detachment should be strongly considered for treatment in the following circumstances: horseshoe tears, large or multiple breaks, dialyses involving more than two ora bays, breaks located posterior to the equator, and breaks in the superotemporal quadrants. Horseshoe tears have continuing vitreous traction because of the invariable attachment of the posterior hyaloid face to the flap of the tear. Posterior breaks are more difficult to treat in the event of retinal detachment because of their location. Retinal breaks in the superior quadrants tend to progress to detachment because of the effect of gravity. Temporal breaks cause retinal detachment because of the effects of gravity. Temporal breaks cause retinal detachments which may pose an early threat to the macula. Breaks with [free] opercula may not require prophylaxis since vitreous traction has been released. Long-standing retinal breaks surrounded by pigmented demarcation lines may be observed at regular intervals."

These observations are as true to-day as when they were written, and are an excellent guide to the management of retinal pathology.

The association of any potentially predisposing retinal lesion with visual symptoms, the discovery of such a condition in the fellow eye of a patient who has had a retinal detachment in the first eye, or the existence of a family history of retinal detachment (Cuendet, 1965) entirely changes the picture. That an incidence of major retinal pathology in the fellow eye occurs in 85 per cent. of cases of spontaneous giant retinal tear is a warning that the most conservative of clinicians must heed. The almost uniform involvement of the fellow eye in retinal detachment after cataract extraction when a similar fate has involved the first eye must lead us seriously to consider prophylactic treatment in the fellow eye before undertaking cataract surgery.

That the risks of spontaneous complications exceed those of prophylactic treatment is becoming well recognized. At the same time, as Cockerham and MacKenzie Freeman (1968) have emphasized, there can be no support for unnecessary nor for excessive treatment. It is only by a critical assessment of methods and their results that the optimal management of retinal problems will be achieved.

According to Rintelen ( 1962 ) and Byer ( 1967 ), the incidence of retinal detachment in a stable population is up to $0^{\circ}$. I per cent., but to the ophthalmologist specializing in retinal surgery this will keep him fully occupied for his working life. For the patient, successful treatment may mean the difference between blindness and the restoration of useful vision.

Prophylactic treatment began with the use of diathermy to seal flat retinal tears, and has progressed, partly as a result of improved instrumentation, to a degree well beyond the sealing of retinal tears by light coagulation or cryotherapy. The observation of multiple retinal lesions in a single eye has prompted the investigation of circumferential treatment by light coagulation or cryotherapy in the hope of isolating the pathological equatorial and pre-equatorial retina from the relatively healthy retina behind the equator.

The possibility of vitreous retraction following a circumferential technique, with the risk of the formation of secondary retinal breaks along the edge of the treated area, or of macular pucker, or of the development of massive vitreous retraction and total (and incurable) retinal detachment, has effectively restricted the use of the method to carefully 
selected cases. Further investigation is still needed accurately to assess the relative merits of prophylactic treatment on the one hand and continued observation on the other. Future work in this field should help us to consolidate our experience. The value of circumferential cryotherapy of the fellow eye in cases of spontaneous giant tear and of aphakic detachment is currently being investigated. It is hoped that it will be possible to demonstrate, in the long term, that this method, carried out in two stages, will provide protection against involvement of the fellow eye without a significant risk of treatment-induced complications.

Surgical encircling procedures in association with cryotherapy or limited light coagulation (Hudson, Kanski, and Elkington, 1973) have been undertaken in cases in which fellow eye involvement has been determined, and in which the first eye has suffered from a retinal detachment associated, in some instances, with massive vitreous retraction. The authors have been strict in their criteria of pre-operative assessment, and are cautiously optimistic about their results.

The principles underlying prophylactic treatment must include the full appreciation of the proper indications. The methods used should provide a maximum degree of protection with a minimum risk of complications. This does not exclude the use of the more sophisticated techniques, but calls upon the experience and clinical judgment of the surgeon specializing in this field.

\section{References}

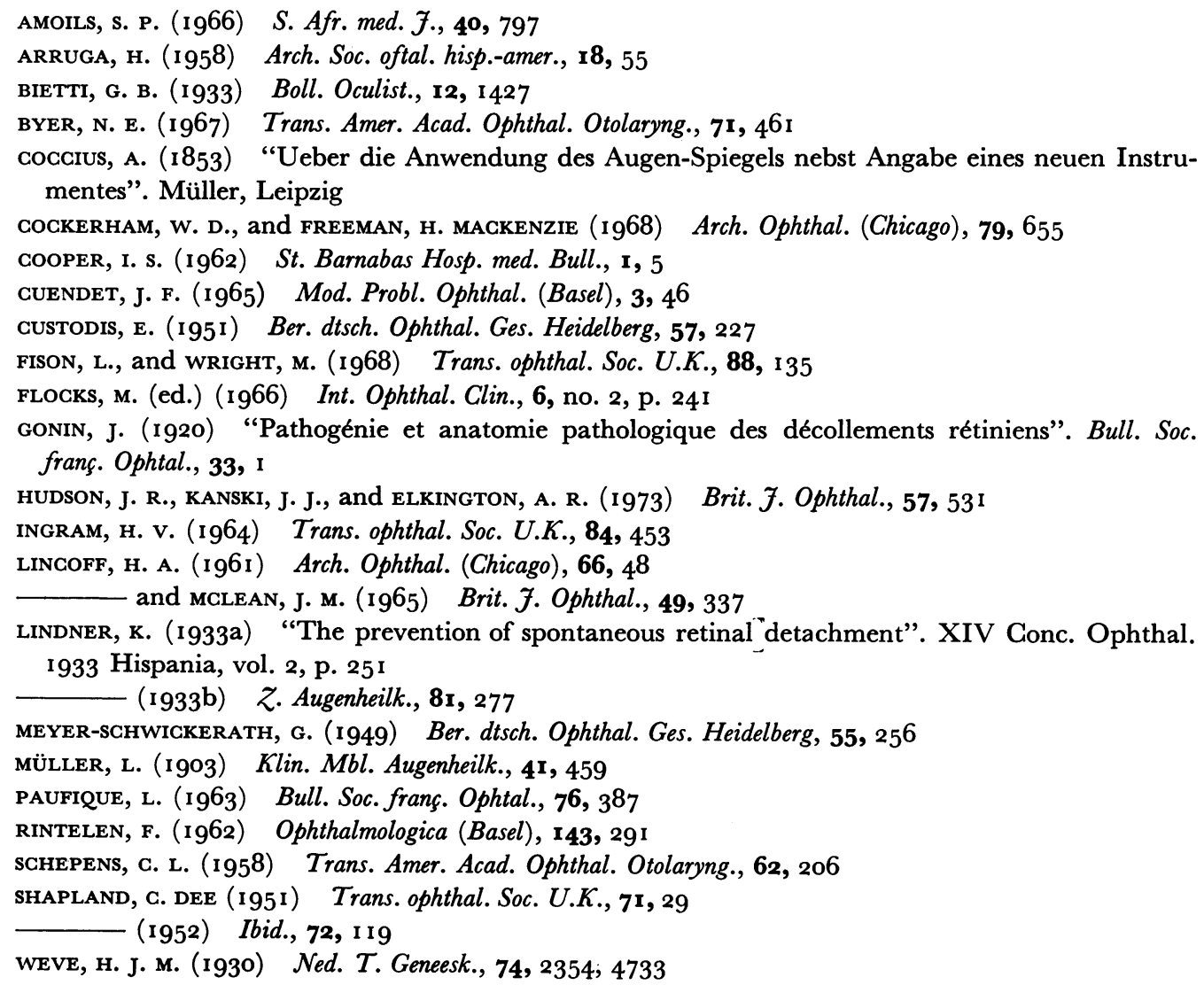

